\title{
Thermoelectric generator fabricated via laser-induced forward transfer
}

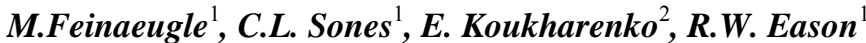 \\ 1. Optoelectronics Research Centre, University of Southampton, Highfield Campus, Southampton, SO17 1BJ, UK \\ 2. School of Physics and Astronomy, ECS, University of Southampton, Highfield Campus, Southampton, SO17 1BJ, UK
}

We show a novel method for the fabrication of a thermoelectric generator with the rapid, lithography-less technique of laser-induced forward transfer (LIFT), performed under ambient conditions. LIFT is a laser-assisted method for the transfer of materials such as metals, semiconductors and dielectrics, where a part of a thin film (donor) previously coated onto a transparent carrier substrate is transferred onto a nearby receiver initiated by the explosive expansion of a small part of the donor volume after the absorption of a laser pulse [1]. Electronic or photonic devices can be fabricated via LIFT on a range of receiver substrates, free from any constraints of substrate properties such as lattice constant or thermal expansion coefficient. This flexibility is desired for applications such as rapid prototyping and the fabrication of devices joining multiple non-standard materials on one substrate. The design of the proposed thermoelectric generator was selected to demonstrate the capabilities of LIFT by transferring layers from the chalcogenide compounds of $\mathrm{Bi}_{2} \mathrm{Te}_{3}$ and $\mathrm{Bi}_{0.5} \mathrm{Sb}_{1.5} \mathrm{Te}_{3}$ onto a glass receiver coated with a polydimethylsiloxane (PDMS) polymer.

For the LIFT-printing of the generator elements, 20ns pulses from a $\mathrm{KrF}$ excimer laser emitting at $248 \mathrm{~nm}$ were used to project a demagnified image of an object aperture onto the interface of the donor and carrier. The elements of the thermoelectric generator were transferred sequentially onto the receiver in order to assemble ntype doped $\left(\mathrm{Bi}_{2} \mathrm{Te}_{3}\right)$ and p-type doped $\left(\mathrm{Bi}_{0.5} \mathrm{Sb}_{1.5} \mathrm{Te}_{3}\right)$ pads that formed a staggered orientation of leg pairs as shown in Fig.1. The donor films of up to $1 \mu \mathrm{m}$ in thickness had been previously sputter-coated onto carriers of fused silica [2]. The staggered design allowed the formation of distinct junctions between the legs which were situated at two opposite edges of the device. When applying a temperature gradient between these opposite sides of the device, the Seebeck effect induced a voltage appearing between the dissimilar elements causing the device to act as a thermoelectric generator.

The Seebeck voltage and the resistance between the thermoelectric elements were measured via a probe station for specific temperature gradients. For a single leg pair, the thermoelectric voltage per unit degree temperature difference was determined to be $>90 \mu \mathrm{V} / \mathrm{K}$. The resistance of a thermoelectric leg pair was in the order of $10 \mathrm{k} \Omega$. The performance was compared to that of thermoelectric generators fabricated both with conventional methods and with devices fabricated with different designs using LIFT..
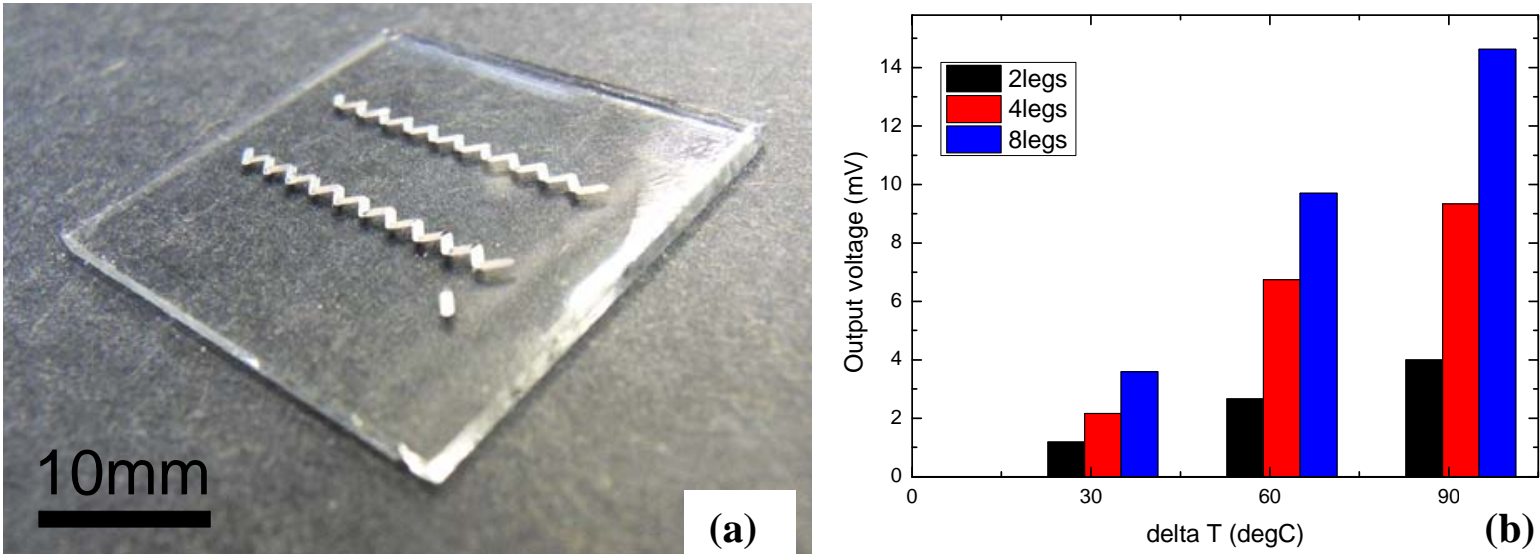

Fig. 1 (a) Picture of two thermoelectric generators on PDMS-coated glass substrate. (b) Output voltage of generators of different sizes depending on the applied temperature gradient.

In conclusion, for the first time, we have demonstrated the printing of a thermoelectric generator via LIFT. The reliability and dependence of successful LIFT-transfer on the laser parameters used and the generator design have been presented. We have also measured the thermoelectric performance of the device to prove its functionality and shown that LIFT is a rapid and novel technique that can be employed for the fabrication of thermoelectric generators on polymer substrate under ambient conditions.

\section{References}

[1] C. B. Arnold, P. Serra, and A. Pique, "Laser direct-write techniques for printing of complex materials," Mrs Bulletin, 32, 1, 23-31, (2007).

[2] M. Feinaeugle, C. L. Sones, E. Koukharenko, B. Gholipour, D. W. Hewak, and R. W. Eason, "Laser-induced forward transfer of intact chalcogenide thin films: resultant morphology and thermoelectric properties," Applied Physics A, (2012). 